\title{
Temperature Compensation for a Microoptical Displacement Sensor Using an Integrated Thermal Sensor
}

\author{
Toshihiro Takeshita, ${ }^{*}$ Yuma Hayashida, ${ }^{1}$ Hideyuki Ando, ${ }^{2}$ \\ Hirofumi Nogami, ${ }^{3}$ and Renshi Sawada ${ }^{3}$ \\ National Institute of Advanced Industrial Science and Technology (AIST), \\ 1-2-1 Namiki, Tsukuba, Ibaraki 305-8564, Japan \\ ${ }^{1}$ Graduate School of Systems Life Sciences, Kyushu University, \\ 744 Motooka, Nishi-Ku, Fukuoka 819-0395, Japan \\ ${ }^{2}$ Fuzzy Logic Systems Institute, 1-5 Hibikino, Wakamatsu-ku, Fukuoka 808-0135, Japan \\ ${ }^{3}$ Department of Mechanical Engineering, Kyushu University, 744 Motooka, Nishi-ku, Fukuoka 819-0395, Japan
}

(Received August 12, 2016; accepted November 25, 2016)

Keywords: optical sensor, displacement sensor, thermal sensor, temperature compensation, piezoelectric actuator

We developed a microoptical displacement sensor on which a thermal sensor was integrated. The sensor was $3 \times 3 \mathrm{~mm}^{2}$ in size and $0.7 \mathrm{~mm}$ thick including the optical power source and thermal sensor. The optical displacement sensor can measure the linear displacement at high resolution without contacting the subject to be measured. We also investigated the dependence of the sensor on thermal change, and we tried to compensate for the dependence when using the thermal sensor by applying the correction functions $A(T), B(T)$, and $C(T)$. These correction functions, which can be easily calibrated, corresponded to the thermal correction of the irradiation angle of the optical power source, the output power of the optical power source, and the sensitivity of photodiodes (PDs), respectively. From the experimental results, the dependence of the optical displacement sensor was reduced from 2.27 to $0.13 \mu \mathrm{m} /{ }^{\circ} \mathrm{C}$. This optical displacement sensor is expected to be used as a position sensor for piezoelectric actuators that also show strong thermal dependence.

\section{Introduction}

With advances in robotics, robots are expected to be used as human assistants in nursing and surgery. In particular, a robot hand is very important in these applications. Robot hands need to be able to grab and hold an object precisely and sensitively. Recently, various kinds of robot hands, actuated by motors, ${ }^{(1,2)}$ a shape-memory alloy, ${ }^{(3)}$ and piezoelectric actuators, ${ }^{(4,5)}$ have been studied and reported. In particular, piezoelectric actuators have many merits, such as high accuracy, high power capacity, and low power consumption. However, their behavior depends greatly on temperature. ${ }^{(6-9)}$ This thermal dependence reduces the accuracy of the displacement of piezoelectric actuators. A piezoelectric actuator may be used with a displacement-enlarging mechanism ${ }^{(5)}$ because the displacement generated by a piezoelectric actuator is very small. As a result, the displacement error caused by a temperature change is also increased. Therefore, the piezoelectric actuator needs to be controlled by a displacement sensor. Displacement sensors, based on principles

${ }^{*}$ Corresponding author: e-mail: toshihiro-takeshita@aist.go.jp 
of capacitance, ${ }^{(10)}$ strain gauge, ${ }^{(11)}$ and others, have been developed and are used to control the displacement of piezoelectric actuators. However, these sensors tend to be large and have a thermal dependence of their own.

We have reported research on a reflective microoptical displacement sensor. ${ }^{(12-14)}$ This sensor consists of a sensor chip on which 12 photodiodes (PDs) are integrated and a vertical-cavity surfaceemitting laser (VCSEL). This sensor is very small, easy to fabricate, and has high resolution. We have already reported embedding the optical displacement sensor into a piezoelectric actuated stage and controlling the displacement error caused by hysteresis using the optical displacement sensor. ${ }^{(12)}$ However, in that report, the thermal dependence of the piezoelectric actuator and the optical displacement sensor were not discussed.

In this paper, we report the structure and characteristics of an optical displacement sensor on which a thermal sensor was integrated. We also investigated the thermal dependence of the optical displacement sensor and a compensation method for its thermal dependence.

One of the novel aspects of this research is the compensation for the change in irradiation angle caused by a temperature change. The characteristics of the optical displacement sensor depend largely on the irradiation angle of the VCSEL. We estimated the change in the irradiation angle of the VCSEL utilizing the feature of the optical displacement sensor in which a number of PDs are integrated on one chip and corrected for its effect on the measured signals. This correction method can not only be used to reduce thermal dependence but also to ensure the reliability and prevent the degradation of the VCSEL.

\section{Materials and Methods}

\subsection{Optical displacement sensor}

Figure 1 shows the structure of the optical displacement sensor. The chip in this sensor is $3 \times$ $3 \mathrm{~mm}^{2}$ in size and $0.7 \mathrm{~mm}$ thick, including the optical power source and thermal sensor. A VCSEL chip is bonded to the center of the sensor chip. The PDs, which are circular with a radius $\left(r_{\mathrm{p}}\right)$ of 60

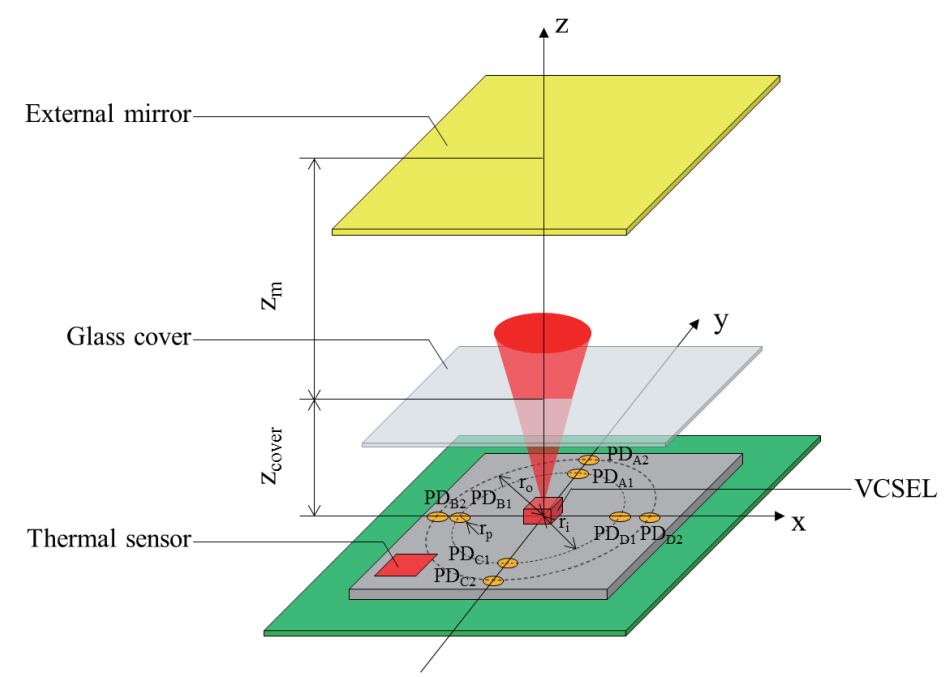

Fig. 1. (Color online) Structure of the optical displacement sensor and thermal sensor. 
$\mu \mathrm{m}$, are placed concentrically with the VCSEL at the center at an inner radius $\left(r_{\mathrm{i}}\right)$ of $650 \mu \mathrm{m}$ and an outer radius $\left(r_{\mathrm{o}}\right)$ of $950 \mu \mathrm{m}$. A thermal sensor was integrated on the chip. The height of the VCSEL is $200 \mu \mathrm{m}$ and the distance from the chip surface to the glass cover, $z_{\text {cover }}$ is $1300 \mu \mathrm{m}$. This optical displacement sensor requires an external mirror as the object to be measured. $z_{\mathrm{m}}$ is the distance from the glass cover to the external mirror.

Figure 2 shows the principle of the optical displacement sensor. After an optical beam is emitted from the VCSEL to the external mirror, it is reflected from the mirror and returns to the surface of the optical displacement sensor. When the mirror moves linearly in the $z$-direction, the beam profile on the surface of the displacement sensor and the outputs of each PD are changed. From the change in outputs, the sensor detects the displacement of the external mirror. The signals for measuring the displacement of the external mirror are defined as Eqs. (1) and (2).

$$
\begin{aligned}
& S_{\text {inner }}=P_{\mathrm{A} 1}+P_{\mathrm{B} 1}+P_{\mathrm{C} 1}+P_{\mathrm{D} 1} \\
& S_{\text {outer }}=P_{\mathrm{A} 2}+P_{\mathrm{B} 2}+P_{\mathrm{C} 2}+P_{\mathrm{D} 2}
\end{aligned}
$$

In these equations, $P_{n}$ is the output of $P D_{n}$, and $S_{\text {inner }}$ and $S_{\text {outer }}$ are the sums of the output signals of the inner four PDs $\left(P D_{\mathrm{A} 1}, P D_{\mathrm{B} 1}, P D_{\mathrm{C} 1}\right.$, and $\left.P D_{\mathrm{D} 1}\right)$ and outer PDs $\left(P D_{\mathrm{A} 2}, P D_{\mathrm{B} 2}, P D_{\mathrm{C} 2}\right.$, and $\left.P D_{\mathrm{D} 2}\right)$, respectively. $S_{\text {inner }}$ is mainly used to measure the displacement. $S_{\text {outer }}$ is used to correct the thermal dependence.

Figure 3 shows the optical displacement sensor integrated with a thermal sensor and an enlarged view of the thermal sensor. There is an Al pad at the center of the sensor chip, and 12 PDs and one thermal sensor are integrated on the chip. We used four inner PDs and four outer PDs and did not
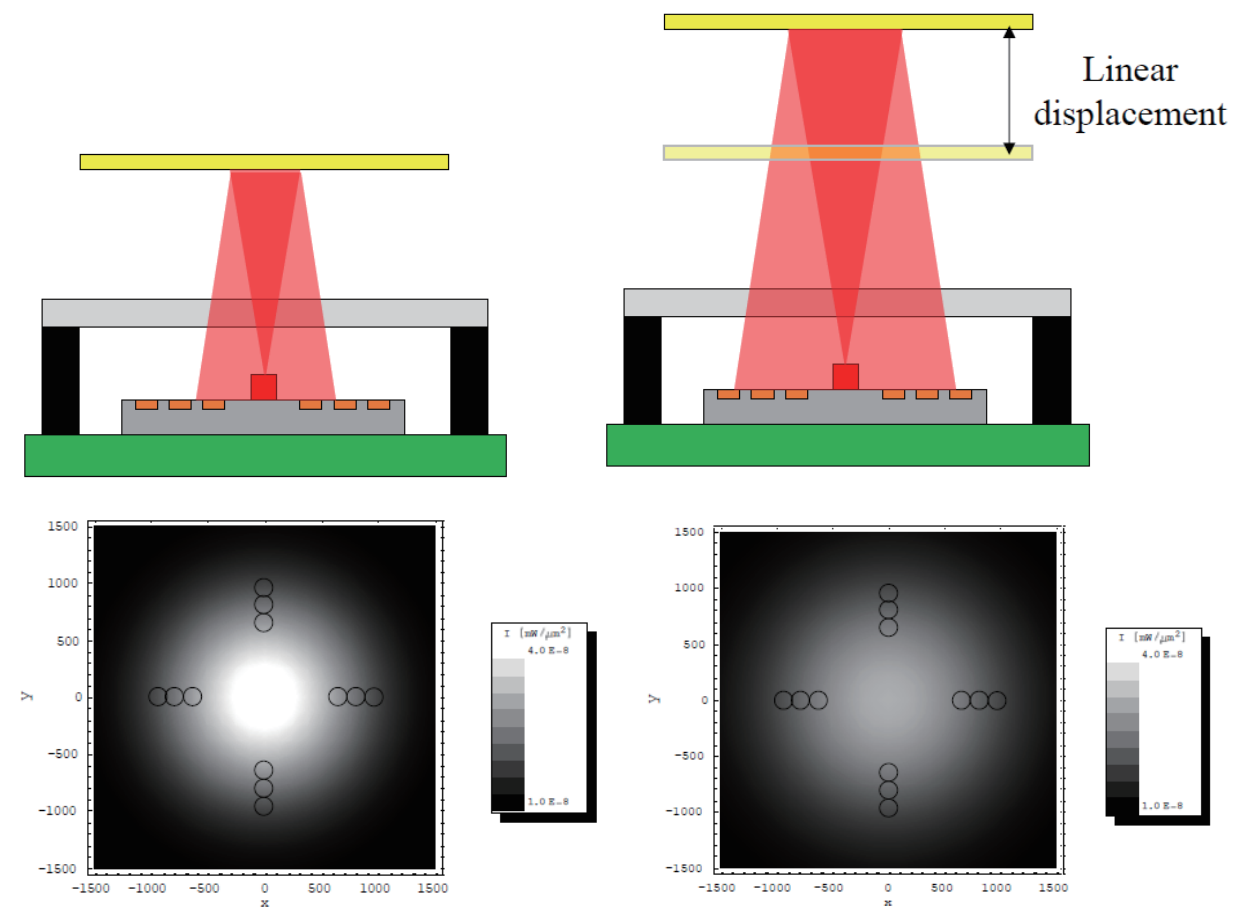

Fig. 2. (Color online) Principle of the optical displacement sensor. 


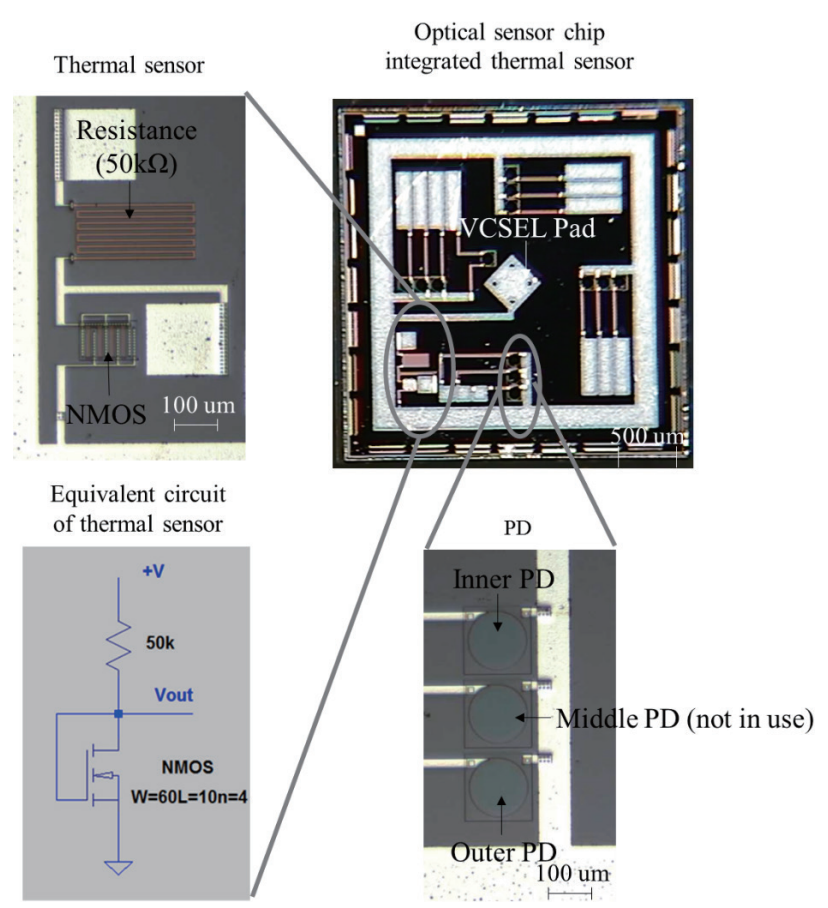

Fig. 3. (Color online) Image of the optical displacement sensor, enlarged view of the thermal sensor, and equivalent circuit of the thermal sensor.

use the middle four PDs in this study. The thermal sensor consisted of one resistor and an NMOS. An equivalent circuit for the thermal sensor is shown in Fig. 3. This thermal sensor detects the difference between the thermal dependence of a resistor and an NMOS. The NMOS has a larger thermal dependence than the resistor, and the divided voltage between the resistor and the NMOS is largely dependent on the thermal change. Therefore, the temperature can be determined by measuring the potential of the divided voltage. A potential of $500 \mathrm{mV}$ was applied on a $+\mathrm{V}$ pad as the power source.

\subsection{Experimental setup}

The experimental setup is shown in Fig. 4. The optical displacement sensor was attached to the jigs. The external mirror, which had a Au surface, was attached to a moving stage. The external mirror was placed in front of the optical displacement sensor. A Peltier heater was placed on the back side of the optical displacement sensor. The heater was connected to a thermal controller. The output voltages of the thermal sensor integrated on the optical sensor chip and the PDs were measured.

First, the characteristics of the thermal sensor were determined in the experiment. Figure 5 shows the output voltage of the thermal sensor integrated on the optical displacement sensor when the heater temperatures were $20,30,40$, and $50{ }^{\circ} \mathrm{C}$. The room temperature was $23{ }^{\circ} \mathrm{C}$. The output voltage depended on the heater temperature and showed good linearity. Therefore, we can estimate the chip temperature from the voltage measurement. 


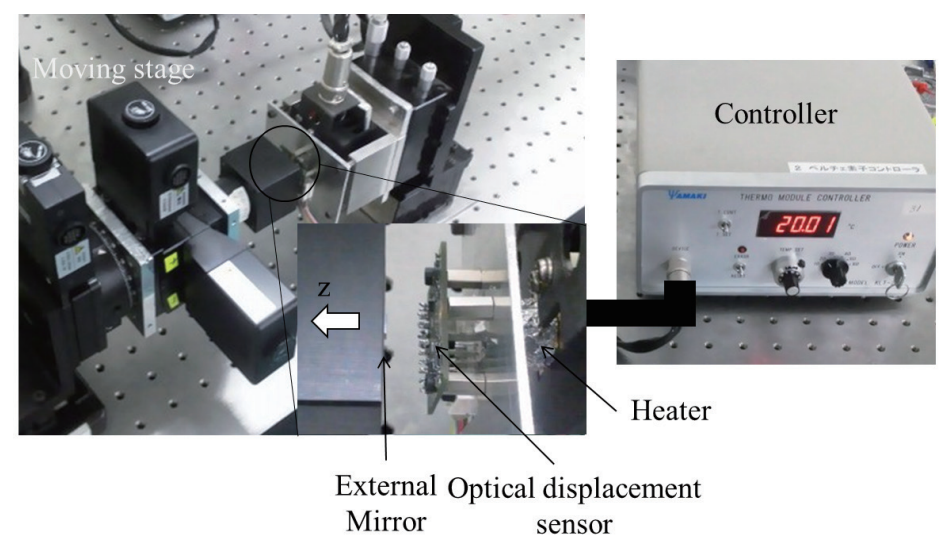

Fig. 4. (Color online) Experimental setup of the optical displacement sensor and the Peltier heater.

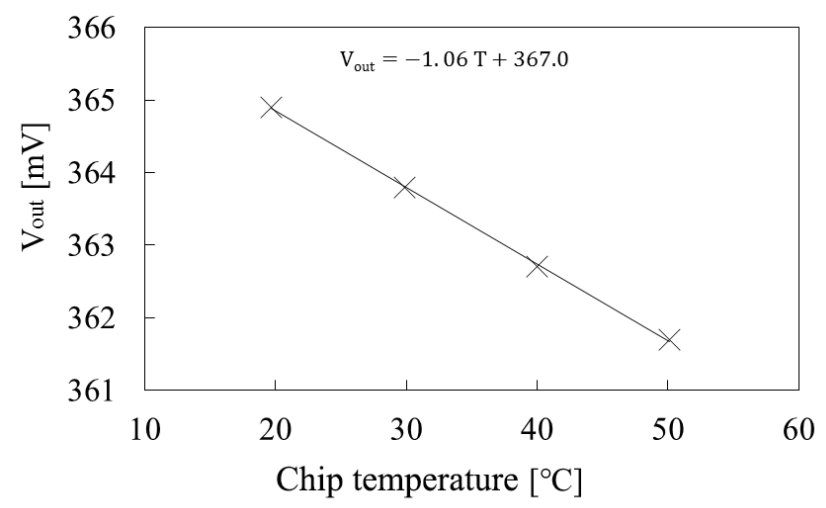

Fig. 5. Experimental results for the thermal sensor, integrated on an optical displacement sensor at temperatures of $20,30,40$, and $50^{\circ} \mathrm{C}$.

The thermal dependence of the optical displacement sensor was determined by measuring the linear displacement of the external mirror. The signals of $S_{\text {inner_T }}$ and $S_{\text {outer_T }}$ were calculated using the output voltage of each PD when the heater temperatures were $20,30,40$, and $50{ }^{\circ} \mathrm{C}$. Figure 6 shows the results. The $S_{\text {inner_T }}$ and $S_{\text {outer_T }}$ showed a large thermal dependence. The thermal dependence of the front slope of $S_{\text {inner_T }}$ was $2.27 \mu \mathrm{m} /{ }^{\circ} \mathrm{C}$, over a measuring range of $100 \mu \mathrm{m}$. The differences in peak positions were caused by a change in the irradiation angle of the VCSEL. When the irradiation angle is smaller, the distance of the external mirror should be larger so that the optical beam is reflected on an external mirror and reaches the PD surface, as shown in Fig. 2. Therefore, when the irradiation angle change is small, the peak positions of the $S_{\text {inner_T }}$ and $S_{\text {outr_T }}$ are shifted too far. Moreover, the differences in the initial and peak values were caused by the output power of the VCSEL and the sensitivity of the PDs.

\section{Thermal Compensation and Results}

We hypothesized that the thermal dependence of the optical displacement sensor was caused by the thermal dependence of the irradiation angle of the VCSEL, the output power of the VCSEL, and the sensitivity of the PDs. Therefore, we defined three correction functions: $A(T)$ for irradiation 


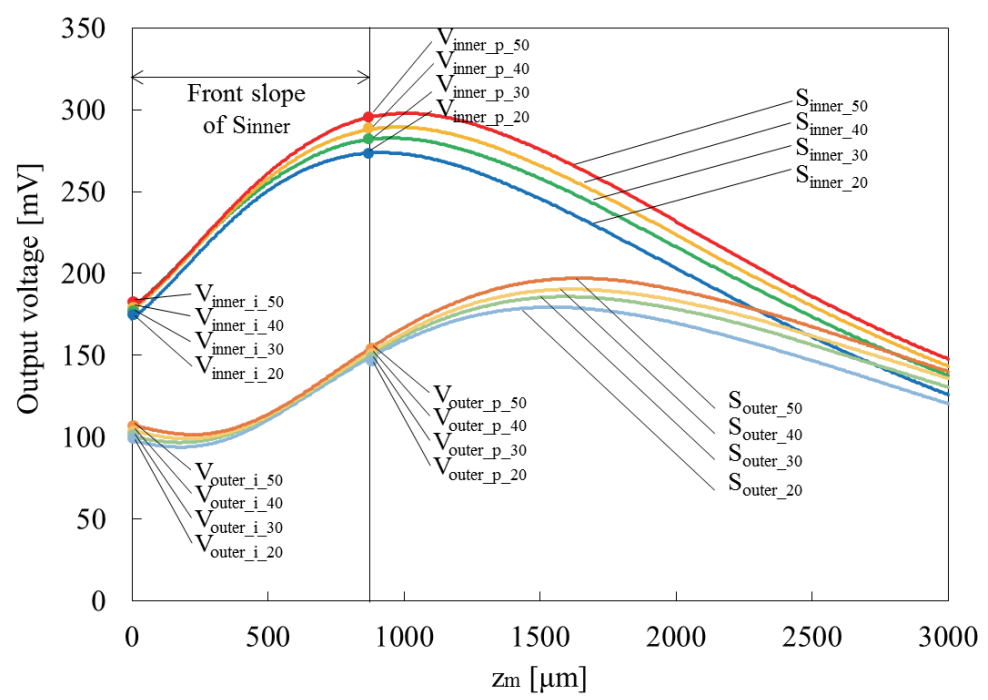

Fig. 6. (Color online) Output signals of $S_{\text {inner_T }}$ and $S_{\text {outer_T }}$ when the external mirror moved from $z_{\mathrm{m}}=0$ to $3000 \mu \mathrm{m}$ and the temperature was $20,30,40$, and $50^{\circ} \mathrm{C}^{-}$.

angle, $B(T)$ for output power, and $C(T)$ for sensitivity, and corrected the thermal dependence using them. T stands for the temperature of the optical displacement sensor and can be measured by the thermal sensor integrated on the optical displacement sensor.

To define a correction function, we measured $S_{\text {inner_T }}$ and $S_{\text {outer_T }}$ at two positons of the external mirror as a calibration step. Table 1 shows the output signals of $S_{\text {inner_T }}$ and $S_{\text {outer_T }}$ when $z_{\mathrm{m}}=0$ and $930 \mu \mathrm{m}$, respectively. When $z_{\mathrm{m}}=930 \mu \mathrm{m}, S_{\text {inner_20 }}$ had a maximum value at $20{ }^{\circ} \mathrm{C}$. The output signals of $S_{\text {inner_T }}$ and $S_{\text {outer_T }}$ when $z_{\mathrm{m}}=0 \mu \mathrm{m}$ are denoted as $V_{\text {inner____ }}$, $V_{\text {outer____ }}$, and those when $z_{\mathrm{m}}=$ $930 \mu \mathrm{m}$ are denoted as $V_{\text {inner___ }}$, and $V_{\text {outer___ }}$, respectively.

\subsection{Definition of $A(T)$}

This sensor is largely affected by the irradiation angle of the optical power source because the radius of the beam spot on the sensor surface is very important for measuring displacement. However, the radius is also changed because of the displacement of the external mirror. In order to separate the effect of the irradiation angle change and the displacement of the external mirror, the irradiation angle change $\left(=\Theta^{\prime}\right)$ is calculated from $V_{\text {inner___T }}, V_{\text {outer_i_T }}, V_{\text {inner___ T }}$, and $V_{\text {outer ___ }}$, under the assumption that the VCSEL has a Gaussian beam profile. The optical power of the VCSEL at the position away from the center of the beam ( $r$ ) is described by Eq. (3).

$$
I(r, z)=\frac{2 P}{\pi z \tan \frac{\Theta}{2}} \exp \left(-\frac{2 r^{2}}{\left(z \tan \frac{\Theta}{2}\right)^{2}}\right)
$$

In this equation, $P, z$, and $\Theta$ are the optical power at the center of the VCSEL, light path length, and beam divergence $\left(1 / e^{2}\right)$ at $20^{\circ} \mathrm{C}$, which is $16.8 \mathrm{deg}$., respectively. When $\Theta$ changes to $\Theta+\Theta^{\prime}$, caused by a change in the temperature, the beam profile is described by Eq. (4). 


$$
I^{\prime}(r, z)=\frac{2 P}{\pi z \tan \frac{\Theta+\Theta^{\prime}}{2}} \exp \left(-\frac{2 r^{2}}{\left(z \tan \frac{\Theta+\Theta^{\prime}}{2}\right)^{2}}\right)
$$

The distances of the inner and outer PDs are $r_{\mathrm{i}}$ and $r_{\mathrm{o}}$, respectively. Therefore,

$$
\begin{gathered}
\log _{e}\left(\frac{I\left(r_{\mathrm{i}}, z\right)}{I\left(r_{\mathrm{o}}, z\right)}\right)=\frac{2 r_{\mathrm{o}}^{2}-2 r_{\mathrm{i}}^{2}}{\left(z \tan \frac{\Theta}{2}\right)^{2}} \\
\log _{e}\left(\frac{I^{\prime}\left(r_{\mathrm{i}}, z\right)}{I^{\prime}\left(r_{\mathrm{o}}, z\right)}\right)=\frac{2 r_{\mathrm{o}}^{2}-2 r_{\mathrm{i}}^{2}}{\left(z \tan \frac{\Theta+\Theta^{\prime}}{2}\right)^{2}} .
\end{gathered}
$$

The term $V_{\text {inner_p_T }}$ corresponds to the optical power at $r=r_{\mathrm{i}}=650 \mu \mathrm{m}, z_{\mathrm{m}}=930 \mu \mathrm{m}$, and at the temperature of $T{ }^{\circ} \mathrm{C}$. The term $V_{\text {outer___ } \mathrm{T}}$ corresponds to the optical power at $r=r_{\mathrm{o}}=950 \mu \mathrm{m}, z_{\mathrm{m}}=$ $930 \mu \mathrm{m}$, and at the temperature of $T^{\circ} \mathrm{C}$. From Eqs. (5) and (6), $\Theta^{\prime}$ is described as Eq. (7).

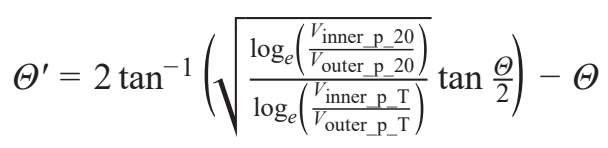

The $\Theta^{\prime}$ values are plotted in Fig. 7 for $T$ equal to $20,30,40$, and $50{ }^{\circ} \mathrm{C}$. A quadratic

\begin{tabular}{|c|c|c|c|c|c|}
\hline & \multirow{2}{*}{$z_{\mathrm{m}}[\mu \mathrm{m}]$} & \multicolumn{4}{|c|}{ Temperature $(\mathrm{T})\left[{ }^{\circ} \mathrm{C}\right]$} \\
\hline & & 20 & 30 & 40 & 50 \\
\hline$V_{\text {inner_i_T }}[\mathrm{mV}]$ & $0(=\mathrm{i})$ & 172.3 & 178.9 & 177.8 & 179.2 \\
\hline$V_{\text {outer_i_T }}[\mathrm{mV}]$ & $0(=\mathrm{i})$ & 98.1 & 100.7 & 104.2 & 107.8 \\
\hline$V_{\text {inner_p_T }}[\mathrm{mV}]$ & $930(=\mathrm{p})$ & 274.2 & 283.5 & 289.7 & 298.9 \\
\hline$V_{\text {outer_p } \mathrm{T}}[\mathrm{mV}]$ & $930(=\mathrm{p})$ & 156.1 & 159.6 & 160.8 & 164.3 \\
\hline
\end{tabular}
approximation curve is defined as Eq. (8).

Table 1

The values of $V_{\text {inner___T }}, V_{\text {outer_i_T }}, V_{\text {inner____ }}$, and $V_{\text {outer___ } \mathrm{T}}$ at temperatures of $20,30,40$, and $50{ }^{\circ} \mathrm{C}$.

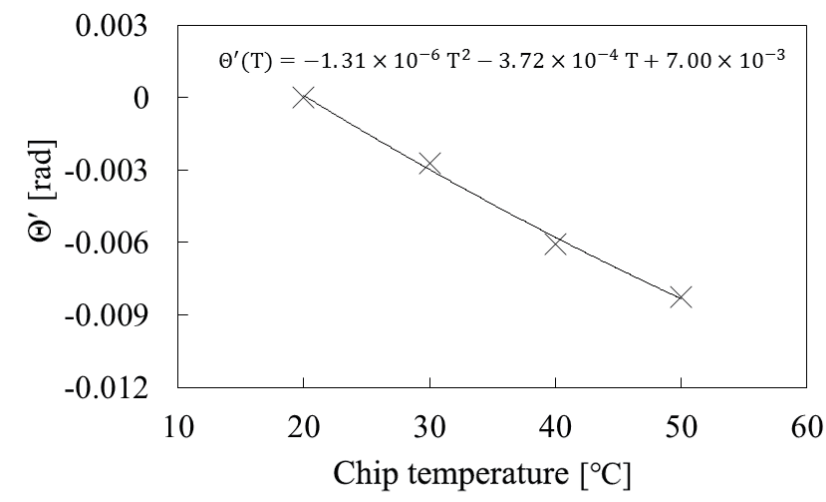

Fig. 7. $\Theta^{\prime}(T)$ calculated using $S_{\text {inner_T }}$ and $S_{\text {outer_T }}$ and the quadratic approximation curve. 


$$
\Theta^{\prime}(T)=-1.31 \times 10^{-6} T^{2}-3.72 \times 10^{-4} T+7.00 \times 10^{-3}
$$

Using Eq. (8), the correct function of $A(T)$ is defined as follows.

$$
A(T)=\frac{I\left(r_{\mathrm{i}}, z\right)}{I^{\prime}\left(r_{\mathrm{i}}, z\right)}=\frac{\tan \left(\frac{\Theta+\Theta^{\prime}(T)}{2}\right)}{\tan \left(\frac{\Theta}{2}\right)} \exp \left(\frac{-2 r_{\mathrm{i}}^{2}}{\left(z \tan \left(\frac{\Theta}{2}\right)\right)^{2}}+\frac{2 r_{\mathrm{i}}^{2}}{\left(z \tan \left(\frac{\Theta+\Theta^{\prime}(T)}{2}\right)\right)^{2}}\right)
$$

Then, $z$, the light path length, is described using $S_{\text {inner T }}$ and $S_{\text {outer_T }}$ from Eqs. (5) and (6) when the temperature is $T$.

$$
z=\sqrt{\frac{-2 r_{\mathrm{i}}^{2}+2 r_{\mathrm{o}}^{2}}{\left(\tan \left(\frac{\Theta+\Theta^{\prime}(T)}{2}\right)\right)^{2} \operatorname{Ln}\left(\frac{S_{\text {mewe } T} T}{\left.S_{\text {out }-\mathrm{T}}\right)}\right.}}
$$

$\Theta, r_{\mathrm{i}}$, and $r_{\mathrm{o}}$ are the structural values of the optical displacement sensor. $\Theta^{\prime}(T)$ is calculated using Eq. (8) and depends on $T$. The $S_{\text {inner_T }}$ and $S_{\text {outer_T }}$ values are the output signals of the inner and outer PDs, respectively, when the temperature is $T^{\circ} \mathrm{C}$. Therefore, $A(T)$ can be determined from the $T$ values, which are estimated using the thermal sensor. Figure 8 shows the $A(T)$ values for $T$ equal to $20,30,40$, and $50^{\circ} \mathrm{C}$. The $A(T)$ values depend on $z_{\mathrm{m}}$, which can be calculated using Eq. (10).

\subsection{Definitions of $B(T)$ and $C(T)$}

Next, we discuss the definitions of $B(T)$ and $C(T)$. To complete the value at the initial position of $S_{\text {inner_T }} \times A(T)$ for each temperature, the constant values were decided and subtracted from the signals. The values are plotted in Fig. 9, and the approximate curve, which was named $B(T)$, is described in Eq. (11).

$$
B(T)=-5.39 \times 10^{-3} T^{2}+0.79 T+161.2
$$

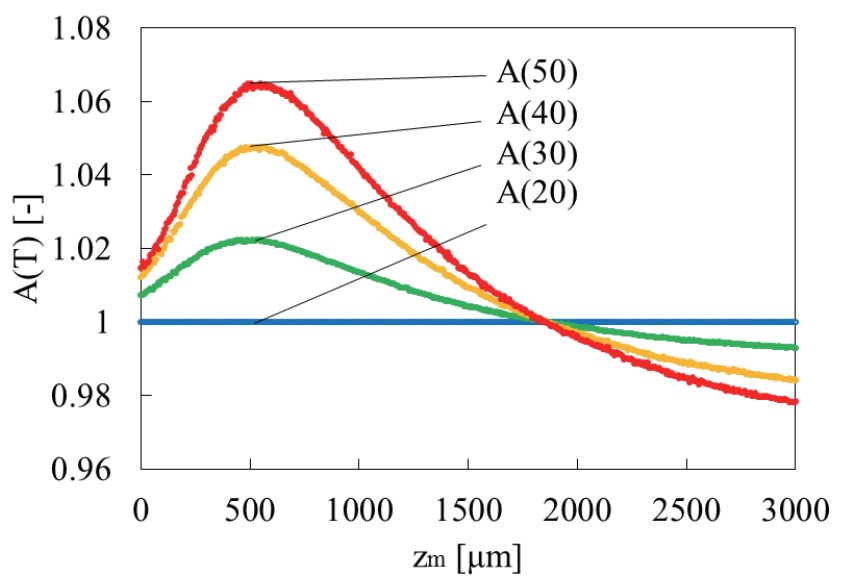

Fig. 8. (Color online) Correction function $A(T)$, calculated using $\Theta^{\prime}(T), S_{\text {inner_- }}$, and $S_{\text {outer_T }}$. 


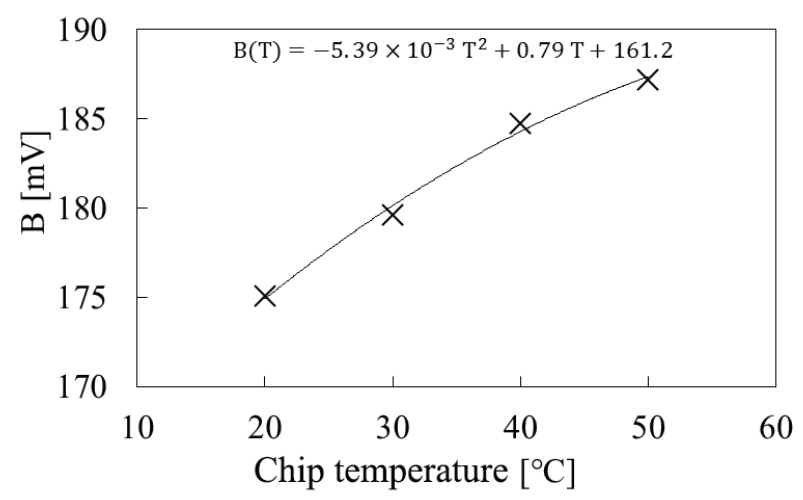

Fig. 9. Quadratic approximation curve and the correction function $B(T)$.

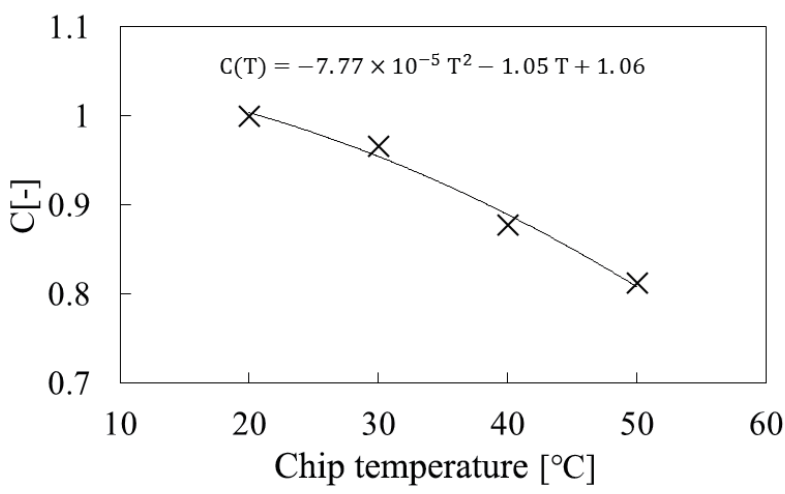

Fig. 10. Quadratic approximation curve and the correction function $C(T)$.

Also, to complete the value $S_{\text {inner_T }} \times A(T)-B(T)$ at the position of $z_{\mathrm{m}}=930 \mu \mathrm{m}$, the constant values were decided and divided from the signals. The values are plotted in Fig. 10, and the approximate curve, which was named $C(T)$, is described in Eq. (12).

$$
C(T)=-7.77 \times 10^{-5} T^{2}+1.05 T+1.06
$$

As described, the correction functions $A(T), B(T)$, and $\mathrm{C}(T)$ are defined as Eqs. (9), (11), and (12), respectively. These correction functions depend on $S_{\text {inner_T }}$ and $S_{\text {outer_ }}$, which can be measured by PDs, and on T, which can be measured by the thermal sensor. Therefore, the values of these functions can be determined at any temperature in the measuring range from 20 to $50{ }^{\circ} \mathrm{C}$, and for any position of the external mirror in the measuring range from $z_{\mathrm{m}}=0$ to $3000 \mu \mathrm{m}$. The term $S_{\text {inner_T }}^{\prime}$, the corrected signal of $S_{\text {inner_T }}$, is defined as Eq. (13).

$$
S_{\text {inner_T }}^{\prime}=\left(S_{\text {inner_T }} \times A(T)-B(T)\right) \times C(T)
$$

\subsection{Results}

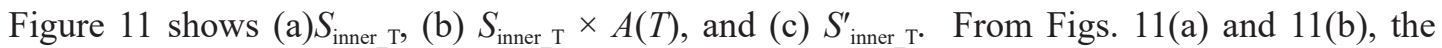
peak positions of each signals were corrected. The difference between the peak positions of $S_{\text {inner_20 }}$ and $S_{\text {inner_50 }}$ was $108 \mu \mathrm{m}$. In contrast, the difference between the peak positions of $S_{\text {inner_20 }}$ $\times A(20)$ and $S_{\text {inner_50 }} \times A(50)$ was reduced to $8 \mu \mathrm{m}$ by $A(T)$. From the results in Fig. 11(c), the thermal dependence at the front slope of $S_{\text {inner_T }}^{\prime}$ was $0.13 \mu \mathrm{m} /{ }^{\circ} \mathrm{C}$, with a measuring range of 100 $\mu \mathrm{m}$. Compared with the thermal dependence of $S_{\text {inner_T }}$, i.e., $2.27 \mu \mathrm{m} /{ }^{\circ} \mathrm{C}$, the value of the thermal dependence was less than $6 \%$ of the previous value.

\section{Discussion}

We now discuss the experimental results. We succeeded in reducing the dependence of the irradiation angle of the VCSEL and reduced the difference in peak positions between $S_{\text {inner_20 }}$ and $S_{\text {inner_50 }}$ from 108 to $8 \mu \mathrm{m}$ using the correction function $A(T)$. The characteristics of the optical 


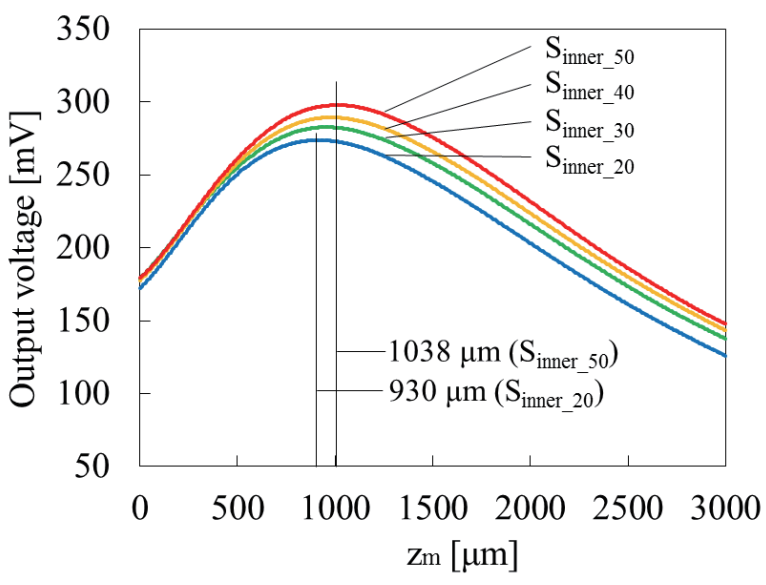

(a)

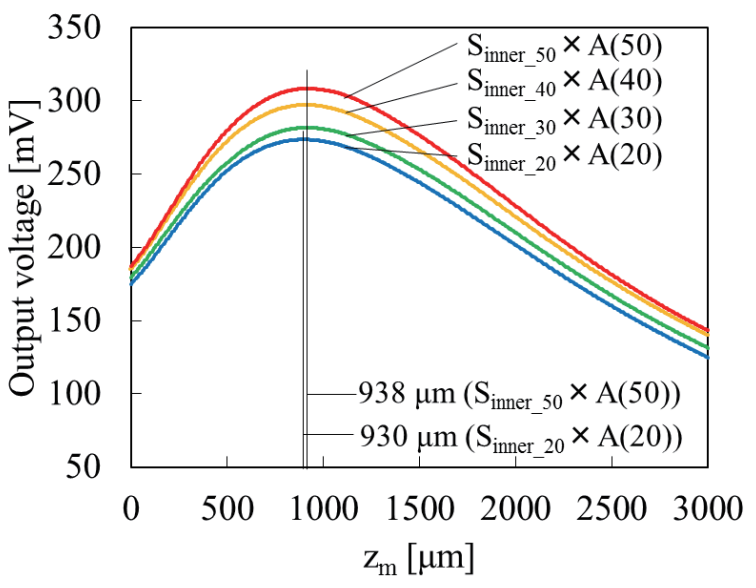

(b)

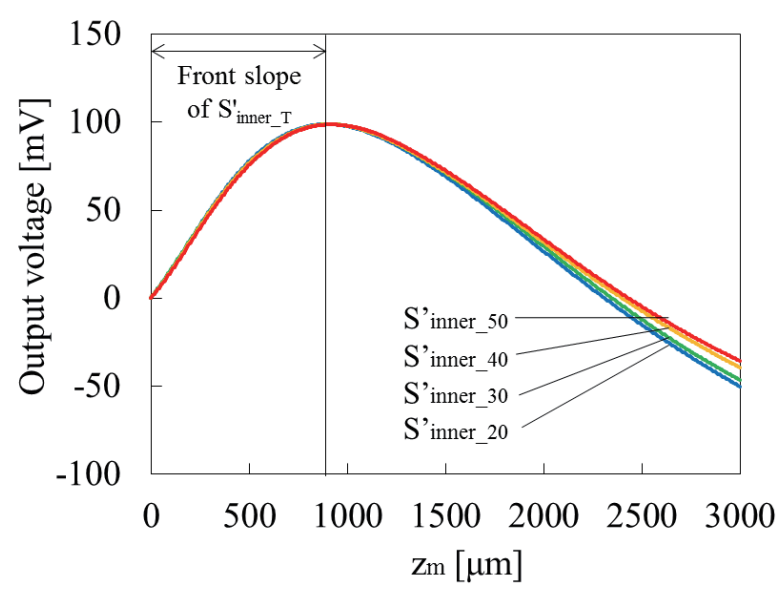

(c)

Fig. 11. (Color online) Experimental results for (a) Sinner_T, (b) Sinner_T $\times$ A(T), and (c) S'inner_T.

displacement sensor are largely dependent on the irradiation angle. Therefore, the correction of irradiation angle is valid not only to reduce thermal dependence but also to ensure the reliability and prevent the degradation of the VCSEL.

Moreover, we succeeded in reducing the effect of thermal change on the signal $S_{\text {inner }}$ at the front slope. The thermal dependence decreased from 2.27 to $0.13 \mu \mathrm{m} /{ }^{\circ} \mathrm{C}$. From Fig. 11(c), it is clear that the signals of $S_{\text {inner }}$ for each temperature were almost the same at the range from the initial position to the peak position. However, these signals were dispersed far from the peak position because of the correction function $A(T)$. The function $A(T)$ is dependent on the signals of $S_{\text {inner_T }}$ and $S_{\text {outer_T. }}$ Particularly, the signal of $S_{\text {outer_T }}$ became smaller as the external mirror moved far from its initial position. As a result, the accuracy of the $A(T)$ value decreased with respect to the peak position. Therefore, the dispersion of the $S_{\text {inner T }}^{\prime}$ signals cannot be removed at positions far from the peak position. This problem may be resolved by adding another correction function defined by the signals at $z_{\mathrm{m}}=3000 \mu \mathrm{m}$ to support the correction function $A(T)$. 


\section{Conclusions}

In this paper, we reported the structure and characteristics of an optical displacement sensor on which a thermal sensor was integrated. The sensor was $3 \times 3 \mathrm{~mm}^{2}$ in size and $0.7 \mathrm{~mm}$ thick, including the optical power source and thermal sensor. We also observed the thermal dependence of the optical displacement sensor and the compensation for the thermal dependence of the signals. The dependence was reduced from 2.27 to $0.13 \mu \mathrm{m} /{ }^{\circ} \mathrm{C}$ using the correction functions $A(T), B(T)$, and $C(T)$.

\section{Acknowledgements}

The device in this study was fabricated at "The 4th Novel Device Design \& Fabrication Contest in Hibikino" held at the Semiconductor Center in Kitakyushu Science and Research Park.

\section{References}

1 P. Dario and G. Buttazzo: Int. J. Rob. Res. 6 (1987) 25.

2 J. K. Salisbury and J. J. Craig: Int. J. Rob. Res. 1 (1982) 4.

3 K. Ikura, D. C. Beard, S. Ho, and H. Moiin: ASME Winter Annual Meeting 14 (1989) 241.

4 S. Wakui: Trans. Jpn. Soc. Mech. Eng. 63 (1997) 2693.

5 Z. Jiang, S. Chonan, and M. Sato: Trans. Jpn. Soc. Mech. Eng. 62 (1996) 920.

6 C. V. Newcomb and I. Flinn: Electron. Lett. 18 (1982) 442.

7 A. Ochi, S. Takahashi, and S. Tagami: J. Appl. Phys. 24 (1985) 209.

8 C. Jer Lin and S. Ren Yang: Mechatronics 16 (2006) 417.

9 S. H. Chang, C. K. Tseng, and H. C. Chien: IEEE Trans. Ultrason. Ferroelectr. Freq. Control 46 (1999) 897.

10 A. E. Holman, W. C. Heerens, and F. Tuinstra: Sens. Actuators, A 36 (1993) 37.

11 A. Furuta, M. Munekara, and T. Higuchi: Jpn. J. Appl. Phys. 41 (2002) 6283.

12 T. Takeshita, T. Iwasaki, K. Harisaki, H. Ando, E. Higurashi, and R. Sawada: Sens. Mater. 26 (2014) 547.

13 T. Takeshita, Y. Peng, N. Morita, H. Ando, E. Higurashi, and R. Sawada: Joint International IMEKO TC1+ TC7+ TC13 Symposium (IMEKO, 2011) urn:nbn:de:gbv:ilm1-2011imeko-015:6.

14 T. Takeshita, K. Harisaki, H. Ando, E. Higurashi, H. Nogami, and R. Sawada: Precis. Eng. 45 (2016) 136. 